\title{
Hydration dependent band gap tunability of self-assembled phenylalanine-tryptophan nanotubes
}

\author{
Hugo A. de Souza Freitas, ${ }^{\dagger}$ Antonio M. J. Chaves Neto, ${ }^{\ddagger}$ Francisco Ferreira de \\ Sousa, ${ }^{\ddagger}$ Rodrigo G. Amorim, "Alexandre Reily Rocha, ${ }^{*, \S}$ and T. Andrade-Filho*,\| \\ $\dagger$ †rograma de Pós-Graduação em Química, Universidade Federal do Sul e Sudeste do Pará, \\ Marabá, PA, Brazil \\ $\ddagger$ Departamento de Física, Universidade Federal do Pará, Belém, Pará, Brazil. \\ IDepartamento de Física, Universidade Federal Fluminense, Volta Redonda, Rio de \\ Janeiro, Brazil. \\ §Instituto de Física Teórica, Universidade Estadual Paulista - UNESP, São Paulo, Brazil. \\ \|Faculdade de Física, Universidade Federal do Sul e Sudeste do Pará, Marabá, Pará, \\ Brazil.
}

E-mail: alexandre.reily@ift.unesp.br; tarcisofilho@unifesspa.edu.br

\section{Abstract}

Bioinspired peptide nanotubes have emerged as a new class of organic semiconductors used in several nanotechnological applications. The long-range order, the trapped solvent and the versatility in the peptide's configuration are some properties that provide an adequate means of tuning the electronic properties of these materials. We investigate the effects of building block separation of PhenylalanineTryptophan nanotube induced by the confined water molecules on the electronic properties using density-functional theory based tightbinding method. The distance between building blocks changes by tuning the amount of confined water. It causes a bandgap modulation of the phenylalanine-tryptophan nanotube (FWNT). All the water-filled structures undergo a transition from an indirect to direct bandgap. Effective control of the electronic properties of PTNTs can allow one to use this 1D-system for bioelectronic medical applications.

\section{Introduction}

Peptides comprise a small chain of amino acids that are the building blocks of proteins, responsible for metabolism in living organisms. ${ }^{1}$ Their self-assembly properties allow us to tailor them - under the right conditions into various motifs. Peptide-based nanostructures such as peptide nanotubes (PNTs), for instance, may lead to a new era of bio-compatible electronic devices. ${ }^{2-5}$ Several factors have contributed to a surge of interest for these systems: they are structurally controllable, almost defect-free, and one can engineer them for specific electronic functions depending on the structural arrangement and choice of peptide. ${ }^{5,6}$ For instance, the diphenylalanine nanotube (FFNT), one of the most well-known representatives of the PNT family, exhibits piezoelectricity, ${ }^{7,8}$ biocompatibility, ${ }^{9}$ high Young modulus, ${ }^{10}$ and outstanding thermal stability. ${ }^{11,12}$

Several works have suggested that most PNTs are semiconductors with wide bandgaps. ${ }^{13,14}$ Carloni et al., for example, calculated the electronic properties of a PNT formed by stack- 
ing octapeptide cyclo $[-(D-A l a-G l u-D-$ Ala $\left.-G l n)_{2}\right]$ units showing that their bandgap was $\sim 4.0 \mathrm{eV} .{ }^{15}$ Sanyal and collaborators predicted, using density functional theory calculations, that nanotubes formed by stacking of $\beta$ cyclic peptides exhibit a similar insulating behaviour; ${ }^{16}$ in the some way that the bandgap in FFNTs was found to be greater than 3.50 eV. ${ }^{17-19}$

If these systems are to be used as electronic devices, however, decreasing the bandgap is required. ${ }^{5}$ One way it can be achieved is via modifications to the driving non-covalent bonds - typically aromatic and hydrogen bonding interactions. ${ }^{5}$ An interesting example to consider is a nanotube-based on the dipeptide phenylalanine-tryptophan $(\mathrm{FW})^{20}$ shown in Figure 1. Akdim et. al. theoretically estimated a reduction of $\sim 1.4 \mathrm{eV}$ in the bandgap of FW nanotube (FWNT) compared to their diphenylalanine counterparts, ${ }^{18}$ and Amdursky et. al. measured a five-fold increase in electrical conductivity just upon replacing diphenylalanine $(\mathrm{FF})$ by a tryptophan amino-acid. ${ }^{21} \mathrm{It}$ is worth mentioning that the tryptophan motif is responsible for charge transfer in biosystems. $^{18}$

In most cases, PNTs are self-organized in an aqueous solution. ${ }^{22}$ It has already been found that water molecules present in a nanotube channel are responsible for stabilizing the zwitterionic structure of each molecule that forms the nanotube. ${ }^{23}$ In particular, depending on the chemical route used for assembly the hydration of the final structure can be tailored. The solvent concentration influences the final structure, ${ }^{24}$ and its electronic properties. ${ }^{19}$

In general, water is detrimental to electronic devices. ${ }^{25}$ The effects of humidity on electronic devices can be significant. In addition to the effects of corrosion, ${ }^{26,27}$ it also induces extreme environmental stress ${ }^{28}$ and swelling in porousbased materials causing structural embrittlement. ${ }^{25-28}$ In possible nanoelectronics applications, it has already been demonstrated that water has the role of isolating the electronic coupling formed between substrate and device. ${ }^{29-31}$ At the same time, we have shown that the presence of water molecules in FFNTs has the dual-effect of stabilizing the structure while reducing the bandgap. ${ }^{17}$

In this work, we report a theoretical investigation on tuning the electronic bandgap of phenylalanine-tryptophan nanotubes by changing the water molecules. Using density functional theory based tight-binding (DFTB) technique with dispersion correction, we show that dehydrated FWNTs present band gap reduction when a single water molecule is embedded. It provides a possible path towards enhanced conductivity be an appropriate selection of the synthesis route.

\section{Methodology}

Tetramers of four FW molecules are used for the assembly of the FW nanotubes. Figure 1 a-b shows a single FW molecule and the corresponding tetramer, which are the building blocks of the anhydrous single FWNT crystal structures (Fig.1 c). The structure resembles diphenylalanine-based ones since it is formed by a hydrophilic central channel with confined solvent molecules and a hydrophobic outer part. In this system, however, the central channel is rectangular. ${ }^{20}$

The starting-point for the NT structure is obtained from x-ray diffraction data. ${ }^{20}$ We obtain the structural and electronic properties by performing self-consistent-charge density-functional tight-binding calculations with a third-order expansion (DFTB3) ${ }^{32}$ using Grimme's dispersion correction (D3). ${ }^{33}$ The pair-interactions for all the calculations are described using the $3 o b$ Slater-Koster parameter set. ${ }^{34,35}$ The Monkhorst-Pack ${ }^{36}$ scheme with a $4 \times 1 \times 1 \mathrm{k}$-point sampling is used, and structural relaxations are carried out until atomic forces are smaller than $0.01 \mathrm{eV} / \AA$. A vacuum region of $\sim 20 \AA$ is left in the lateral directions to avoid spurious inter-tube interactions.

The simulations start with the relaxation of the anhydrous single FWNT crystal structure (final relaxed geometry shown in Fig.1). We consider up to three water molecules per tetramer, inserted in the channel using a box-constrained algorithm implemented in the 


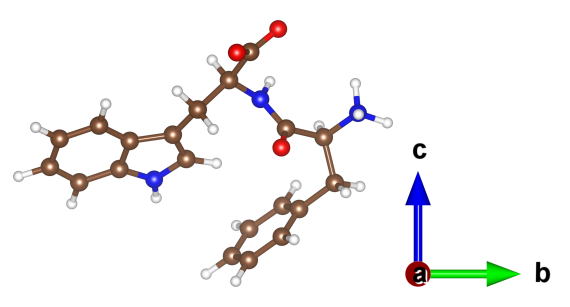

a)

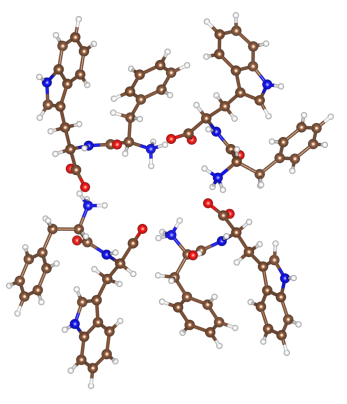

b)

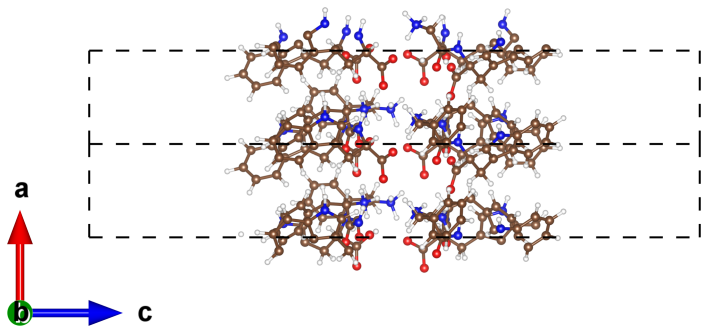

c)

Figure 1: (Color online) a) A single FW molecule, b) tetramer building-block unit of four FW molecules forming a ring and c) vertical stacking of tetramer units forming a single NT.

Packmol program. ${ }^{37,38}$

Finally, one uses the structures as input to DFTB3-MD simulations via a simulated annealing protocol. ${ }^{39}$ We carry out the calculations in the NVT Ensemble using the Andersen thermostat $^{40}$ and the ionic equations of motion are solved using the velocity-verlet algorithm via a time integration step of 0.5 fs. The structures are heated from 0 to $300 \mathrm{~K}$ during $5.0 \mathrm{ps}$, and they are then kept at room temperature for an extra $10.0 \mathrm{ps}$. After that, the systems are cooled to $0 \mathrm{~K}$ in $5.0 \mathrm{ps}$. All the calculations are performed using the DFTB + code $^{41}$

\section{Results and discussion}

For the water-free FWNT geometry, we obtain a lattice parameter of $5.42 \AA$. Our result represents a compression of approximately $3.7 \%$ when compared to experimental values with NTs containing three water molecules per tetramer. ${ }^{20}$ In Table 1, we present the evolution of lattice constant as a function of the number of water molecules inside the FWNT. Interestingly, the lattice constant shows oscillatory behaviour which indicates an interplay between non-bonding interactions with water, and steric effects.

The bandstructures for all systems that are considered are shown in Fig. 2. We note a direct correlation between the changes in bandgap to variations in the lattice parameter $a$, i.e. the magnitude of the bandgap de- pends directly on the tetramer distance induced by the number of confined water molecules. It is worth mentioning that the anhydrous FWNT bandgap compares well with DFT calculations performed with hybrid exchangecorrelation functionals (HSE06). ${ }^{18}$ Thus, one can conclude that the bandgap increases with increasing the stacking distance. The opposite also occurs: the band gap decreases with the stacking distance during water molecule removal (Fig. 2). It is worth mentioning that the FWNT bandgap tuning behaviour dependent on the stacking and the concentration of confined water molecules is similar to the one observed by the stacking of few-layer boronphosphide. ${ }^{42}$

Table 1: Structural and electronic properties for FWNT with different hydration: bandgap, the lattice constant along the NT axis, and coupling energy.

\begin{tabular}{|cc|cc|}
\hline$\# \mathrm{H}_{2} \mathrm{O}$ molecules & $a(\AA)$ & Band gap $(\mathrm{eV})$ & $\mathrm{E}_{\text {coup }}(\mathrm{eV})$ \\
\hline 3 & 5.42 & 3.27 & -0.66 \\
\hline 2 & 5.48 & 3.33 & -0.66 \\
\hline 1 & 5.34 & 2.85 & -0.33 \\
\hline 0 & 5.37 & 2.96 & - \\
\hline Exp. $^{20}$ & 5.63 & - & - \\
\hline
\end{tabular}

Analyzing which tube section contributes to the electronic states with three and two confined water molecules one can note that the top of the valence band $(V B)$ and the minimal of the conduction band $(C B)$ are ruled by $\mathrm{C}$ (in- 
a)

b)

c)

d)
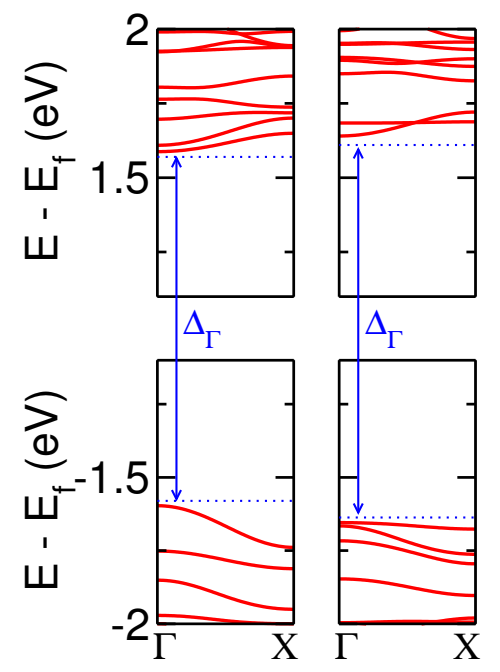

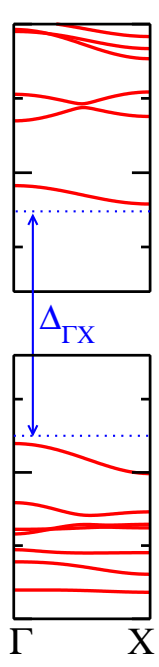

Figure 2: (Color online) Band structure of the FWNT containing a) $3 \mathrm{H}_{2} \mathrm{O}$, b) $2 \mathrm{H}_{2} \mathrm{O}$, c) $1 \mathrm{H}_{2} \mathrm{O}$ and d) the water-free case.

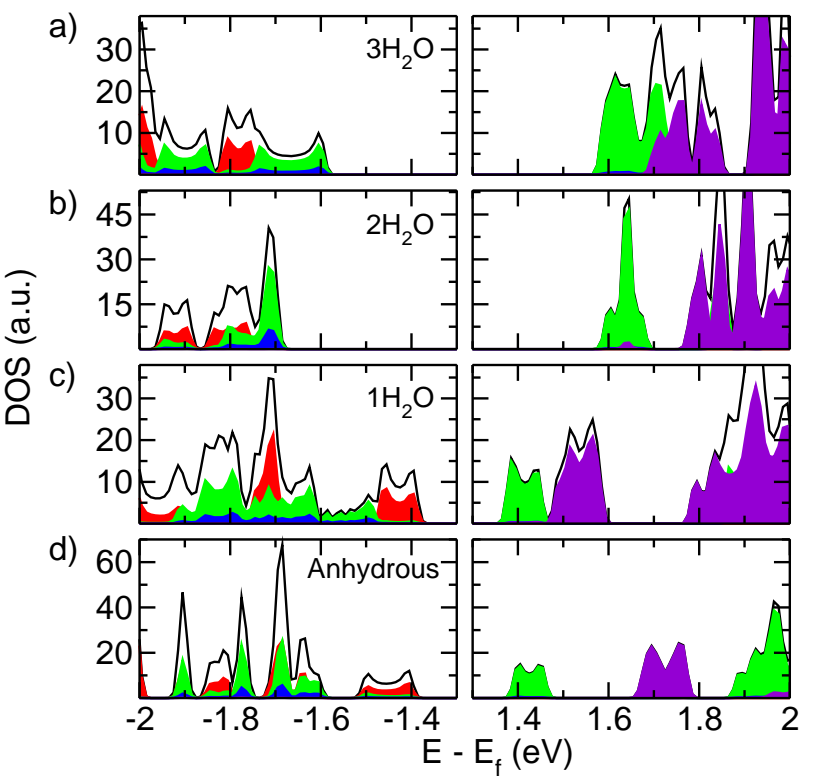

Figure 3: (Color online) Total and orbital projected density of states of the FWNT with 1-3 water molecule and the anhydrous case. Red, green, blue, and purple lines refer to $2 \mathrm{p}$ states of carboxylate oxygens, indole carbons, indole nitrogens and aromatic carbons, respectively. The black line corresponds to the total density of states.

dole group) $2 p$ states at the FW molecules. It may be visible from the projected density of states shown in Fig. 3. If we compare with the system without confined water molecules, we observe that the edge of the valence band and the conduction band of the last two systems shift downwards and upwards, respectively. As a result, we notice an increase in bandgap. For one water molecule trapped in the tube, the edge of the $\mathrm{VB}$ is formed mainly by $\mathrm{O}\left(\mathrm{COO}^{-}\right)$ $2 p$ states. On the other hand, the edge of the $\mathrm{CB}$ states is formed by the $\mathrm{C}$ (indole group) and $\mathrm{C}$ (aromatic groups) 2p states. The distribution of states at the edge of the valence and conduction bands for the water-free nanotube is similar for the case that contains a single confined water molecule.

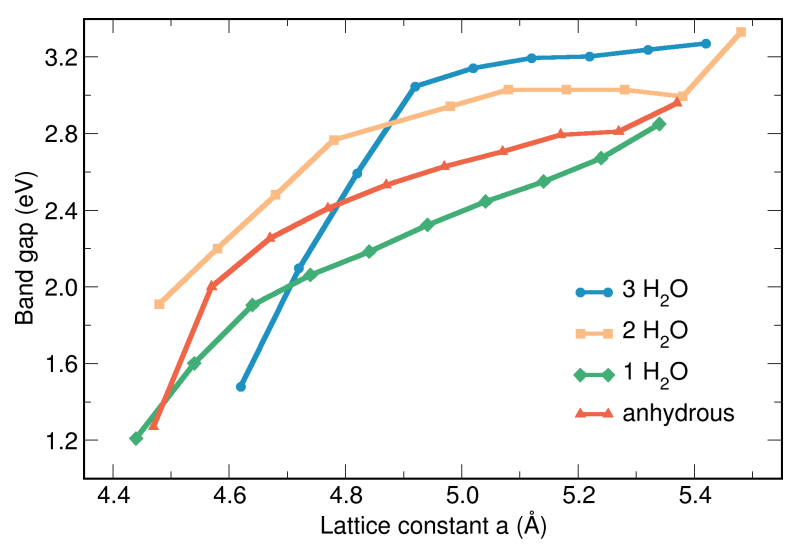

Figure 4: (Color online) Evolution of the band gaps of the FWNT containing a) 3, b) 2, c) 1 water molecules and d) the anhydrous case as a function of the stacking distance.

With three and two water molecules per tetramer, one notes three peaks between -3.70 and $-1.91 \mathrm{eV}$ belonging to bands of water oxygen $2 \mathrm{p}$ states. One describes water $\mathrm{O} 2 \mathrm{p}$ states by five peaks between -3.60 and $-1.83 \mathrm{eV}$ for the remaining system (not shown). Based on the above results, we find that water molecules play an important role in tuning the electronic structure of the FWNT. In the calculations, the complex containing a single water molecule shows the most prominent semiconductor character, followed by the bare nanotube. One notes an insulator behavior for the other configurations.

We analyze how the bandgap varies with stacking distance. In Fig. 4 we show the evo- 

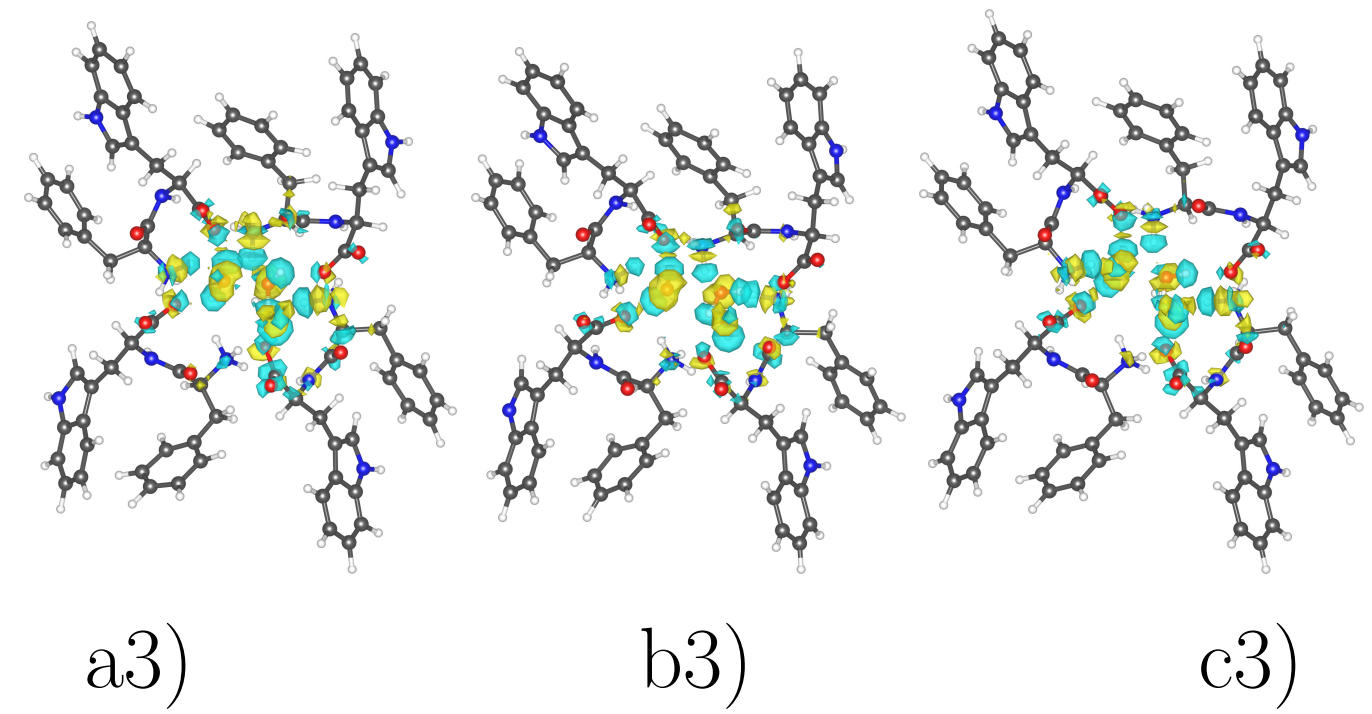

b3)

c3)
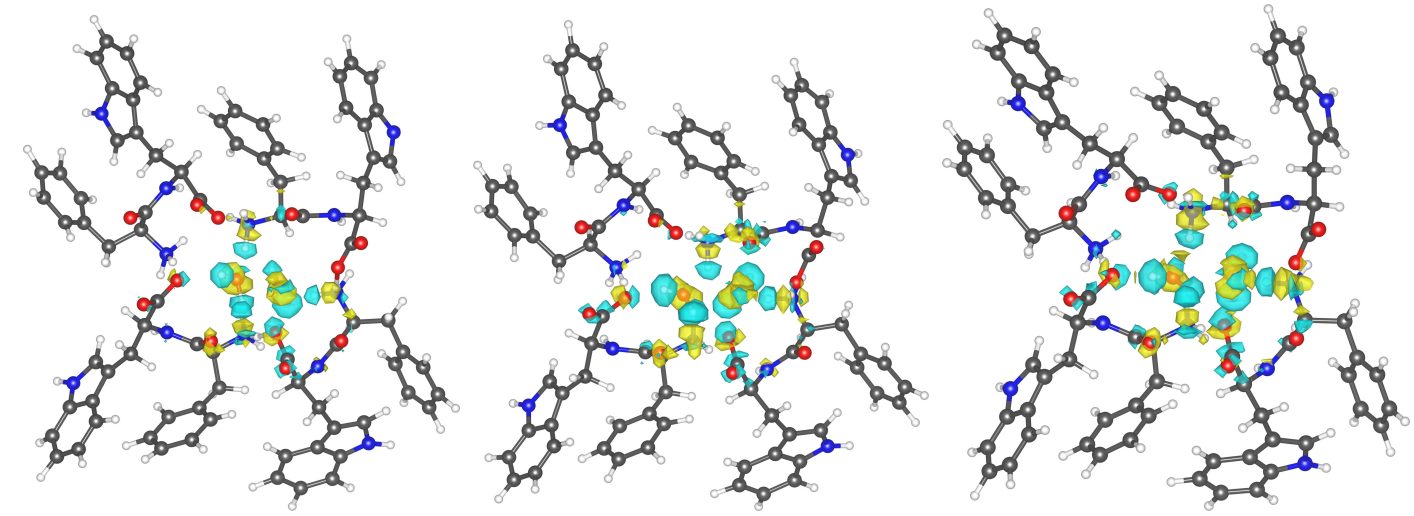

a2)

b2)

c2)

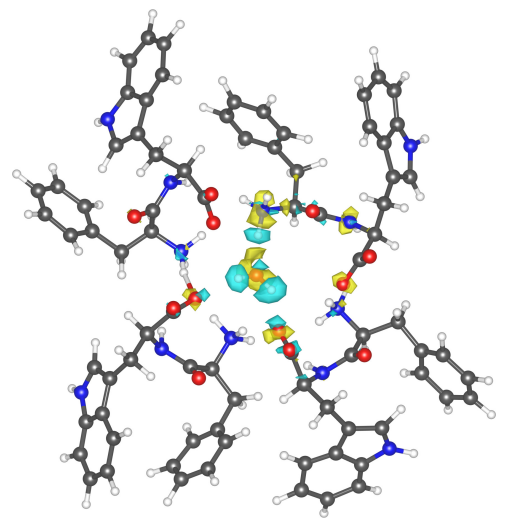

a1)

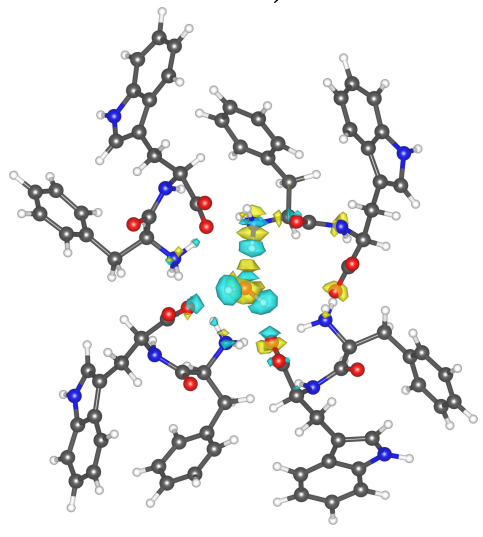

b1)

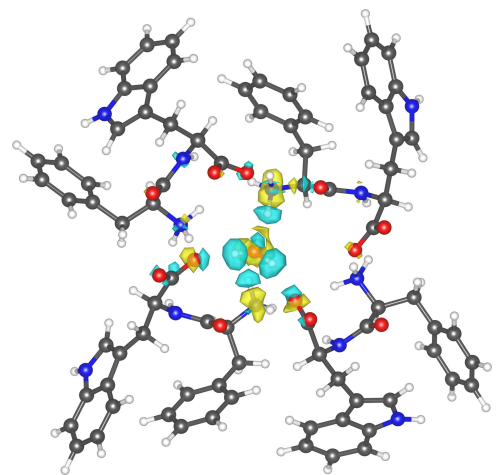

C1)

Figure 5: (Color online) Charge density difference isosurfaces of FWNT with 3 (a1, b1 and c2), 2 (a2, b2 and $\mathrm{c} 2$ ) and 1 (a3, b3 and c3) confined water molecules. They are obtained at 5.00 ps (a), $15.00 \mathrm{ps}$ (ps) and $20.00 \mathrm{ps}$ (c). Yellow and green colors represent charge depletion and accumulation, respectively. The isovalue is set to $\pm 0.02 \mathrm{e}^{-3}$. 
lution of the bandgaps of the FWNT containing a) 3, b) 2, c) 1 water molecules and d) the water-free case as a function of the stacking distance. One can note in this figure the almost linear character of the bandgap concerning the stacking distance for the anhydrous and $1 \mathrm{H}_{2} \mathrm{O} @ \mathrm{FWNT}$ systems. That is, we can achieve bandgap reduction via way of means of decreasing the stacking distance for those systems. Even with the stacking distances reduced to approximately $4.7 \AA$, the water molecules of the $2 \mathrm{H}_{2} \mathrm{O} @ \mathrm{FWNT}$ system keep the bandgap almost constant. After a slight reduction because of the decrease in the lattice constant $a$, we note that the water molecules of the $3 \mathrm{H}_{2} \mathrm{O} @ \mathrm{FWNT}$ system keep the bandgap almost constant until $4.9 \AA$. For these last two systems, it is worth mentioning that the bandgap's almost linear behavior concerning the stacking distance when reducing the mentioned cutoff distances. According to these results, we conclude that the combined effect of stacking distance and water concentration leads the phenylalaninetryptophan nanotube to behave as a semiconductor / an insulator system.

To analyze the interaction energy between the FWNT and water molecules, one evaluates the coupling energy $\left(\mathrm{E}_{C}\right)$. It is given by the difference in energy between the complex $\left(\mathrm{FWNT}+\mathrm{H}_{2} \mathrm{O}\right)$ and the energies of the isolated nanotube and corresponding confined water chain. ${ }^{17}$ We normalize all calculations by the number of water molecules and present them in Table 1. In general, water molecules strongly bind to the nanotube hydrophilic channel. One can attribute the high coupling energy value to the hydrogen bond formation between tube and molecules. In that sense, the strong interaction with the water molecules induces the modification in the electronic structure of the system.

The charge density redistribution of different MD configurations is shown in Fig. 5. It is defined as:

$$
\Delta \rho=\rho_{P T N T+H_{2} O}-\left(\rho_{P T N T}+\rho_{H_{2} O}\right)
$$

where $\rho_{P T N T+H_{2} O}, \rho_{P T N T}$, and $\rho_{H_{2} O}$ are the charge densities of the complex, isolated nan- otube and water molecules, respectively. It can be used to provide information concerning the charge redistribution in the system due to the water. One can note hydrogen bond formation from the charge accumulation region on the water, the carboxylate oxygen, and some ammonium nitrogen atoms and the charge depletion region on the water and ammonium hydrogen atoms. The charge accumulation lobes are larger for cases with 3 and 2 confined water molecules leading to the behavior of the coupling energy. ${ }^{43}$

The biocompatibility, bio-recognition, and tunable band gaps between anhydrous and water-trapped FWNT give rise to potential bioelectronic applications since it is possible to control the removal of water molecules confined within this nanotube. ${ }^{20,21,44}$

\section{Conclusions}

According to what we observe in this work, it is possible to relate bandgap tuning to a combination of stacking distance and water concentration. One finds the band structure to be more dispersive than the ones calculated for the diphenylalanine nanotubes. It results from the significantly tighter packing of the four FW molecules that compose the building block of the NT. ${ }^{45}$ In consonance with the calculated electronic band structures, one can determine that the FWNT structure can behave as semiconductors. ${ }^{18}$ Besides other properties such as biocompatibility and bio-recognition, the possibility of having a semiconductor system with a tunable bandgap suggests its use in bioelectronic medical applications. ${ }^{44}$

Acknowledgement This study is financed in part by the Coordenação de Aperfeiçoamento de Pessoal de Nível Superior - Brasil (CAPES), and Brazilian Institute of Science and Technology (INCT) in Carbon Nanomaterials, CNPq and FAPEMIG. The financial support of the Brazilian National Council for Scientific and Technological Development (CNPq) Brazilian agency under project Universal (grant $427527 / 2016-3)$, is also gratefully acknowledged. A.R.R. acknowledges support from the 
ICTP-Simons Foundation Associate Scheme, and R.G.A. acknowledges financial support from CNPq (2535/2017-1 and 437182/20185). The authors also thank LCC/UNIFESSPA for the computation facilities made available for this work.

\section{References}

(1) Brayden, D. J. Evolving peptides for oral intake. Nature Biomed. Eng. 2020, 4, 487.

(2) Panda, S. S.; Katz, H. E.; Tovar, J. D. Solid-state electrical applications of protein and peptide based nanomaterials. Chem. Soc. Rev. 2018, 47, 3640.

(3) Jenkins, K.; Kelly, S.; Nguyen, V.; Wu, Y.; Yang, R. Piezoelectric diphenylalanine peptide for greatly improved flexible nanogenerators. Nano Energy 2018, 51, 317.

(4) Ing, N. L.; Spencer, R. K.; Luong, S. H.; Nguyen, H. D.; Hochbaum, A. I. Electronic Conductivity in Biomimetic Helical Peptide Nanofibers and Gels. ACS Nano 2018, 12, 2652.

(5) Tao, K.; Makam, P.; Aizen, R.; Gazit, E. Self-assembling peptide semiconductors. Science 2017, 358, eaam9756.

(6) Tsai, C. J.; Zheng, J.; Nussinov, R. Designing a Nanotube Using Naturally Occurring Protein Building Blocks. PLoS Comput. Biol. 2006, 2, e42.

(7) Nguyen, V.; Zhu, R.; Jenkins, K.; Yang, R. Self-assembly of diphenylalanine peptide with controlled polarization for power generation. Nature Commun. 2016, 7, 13566.

(8) Safaryan, S.; Slabov, V.; Kopyl, S.; Romanyuk, K.; Bdikin, I.; Vasilev, S.; Zelenovskiy, P.; Shur, V. Y.; Uslamin, E. A.; Pidko, E. A. et al. Diphenylalanine-Based Microribbons for Piezoelectric Applications via Inkjet Printing. ACS Appl. Mater. Inter. 2018, 10, 10543.
(9) Feyzizarnagh, H.; Yoon, D.; Goltz, M.; Kim, D. Peptide nanostructures in biomedical technology. WIREs Nanomed. Nanobiotechnol. 8, 730 .

(10) Kol, N.; Adler-Abramovich, L.; Barlam, D.; Shneck, R. Z.; Gazit, E.; Rousso, I. Self-Assembled Peptide Nanotubes Are Uniquely Rigid Bioinspired Supramolecular Structures. Nano Lett. 2005, 5, 1343.

(11) Adler-Abramovich, L.; Reches, M.; Sedman, V. L.; Allen, S.; Tendler, S. . J. B. .; Gazit, E. Thermal and Chemical Stability of Diphenylalanine Peptide Nanotubes:Implications for Nanotechnological Applications. Langmuir 2006, 22, 1313.

(12) Andersen, K. B.; Castillo-Leon, J.; Hedström, M.; Svendsen, W. E. Stability of diphenylalanine peptide nanotubes in solution. Nanoscale 2011, 3, 994.

(13) Ashkenasy, N.; Horne, W. S.; Ghadiri, M. R. Design of Self-Assembling Peptide Nanotubes with Delocalized Electronic States. Small 2006, 2, 100.

(14) Takahashi, R.; Wang, H.; Lewis, J. P. Electronic Structures and Conductivity in Peptide Nanotubes. J. Phys. Chem. B 2007, 111, 9093.

(15) Carloni, P.; Andreoni, W.; Parrinello, M. Self-Assembled Peptide Nanotubes from First Principles. Phys. Rev. Lett. 1997, 79, 761.

(16) Sanyal, B.; Eriksson, O.; Arvidsson, P. I. Cobalt-doped $\beta$-peptide nanotubes: A class of spintronic materials. Phys. Rev. B 2008, 77, 155407.

(17) Andrade-Filho, T.; Ferreira, F. F.; Alves, W. A.; Rocha, A. R. The effects of water molecules on the electronic and structural properties of peptide nanotubes. Phys. Chem. Chem. Phys. 2013, 15,7555 . 
(18) Akdim, B.; Pachter, R.; Naik, R. R. Self-assembled peptide nanotubes as electronic materials: An evaluation from firstprinciples calculations. Appl. Phys. Lett. 2015, 106, 183707.

(19) Wang, M.; Xiong, S.; Wu, X.; Chu, P. K. Effects of Water Molecules on Photoluminescence from Hierarchical Peptide Nanotubes and Water Probing Capability. Small 2011, 7, 2801.

(20) Gorbitz, C. H. The structure of nanotubes formed by diphenylalanine, the core recognition motif of Alzheimers amyloid polypeptide. Chem. Commun. 2006, 2332.

(21) Amdursky, N. Enhanced solid-state electron transport viatryptophan containing peptide networks. Phys. Chem. Chem. Phys. 2013, 15, 13479.

(22) Xia, J.; Yang, P.; Sun, Y.; Wu, Y.; Mayers, B.; Gates, B.; Yin, Y.; Kim, F.; Yan, H. One-Dimensional Nanostructures: Synthesis, Characterization, and Applications. Adv. Mater. 2003, 15, 353.

(23) Andrade-Filho, T.; Martins, T. C.; Ferreira, F. F.; Alves, W. A.; Rocha, A. R. Water-driven stabilization of diphenylalanine nanotube structures. Theor. Chem. Acc. 2016, 135.

(24) Kim, J.; Han, T. H.; Kim, Y. I.; Park, J. S.; Choi, J.; Churchill, D. G.; Kim, S. O.; Ihee, H. Role of Water in Directing Diphenylalanine Assembly into Nanotubes and Nanowires. Adv. Mater. 2010, 22, 583.

(25) Isecke, B.; Schütze, M.; Strehblow, H. H. Springer Handbook of Metrology and Testing; Springer Berlin Heidelberg, 2011; p 667.

(26) Comizzoli, R. B. Corrosion and Reliability of Electronic Materials and Devices: Proceedings of the Second International Symposium, Vol 93-1 (Proceedings / Electro- chemical Society); Electrochemical Society, 1993.

(27) Ohring, M.; Kasprzak, L. Reliability and Failure of Electronic Materials and Devices, 2nd ed.; Elsevier, 2011.

(28) Cressler, J. D.; Mantooth, H. A. Extreme Environment Electronics (Industrial Electronics); CRC Press, 2017.

(29) Wang, Y.; Xu, Z. Water Intercalation for Seamless, Electrically Insulating, and Thermally Transparent Interfaces. ACS Appl. Mater. Int. 2016, 8, 1970.

(30) Banerjee, G. K. Electrical and Electronics Engineering Materials; Prentice-Hall of India Pvt.Ltd, 2015.

(31) Hakeem, K. R.; Jawaid, M.; Rashid, U. Biomass and Bioenergy: Applications; Springer, 2014.

(32) Gaus, M.; Cui, Q.; Elstner, M. DFTB3: Extension of the Self-Consistent-Charge Density-Functional Tight-Binding Method (SCC-DFTB). J. Chem. Theory Comput. 2011, \%, 931.

(33) Grimme, S.; Antony, J.; Ehrlich, S.; Krieg, H. A consistent and accurate ab initio parametrization of density functional dispersion correction (DFT-D) for the 94 elements H-Pu. J. Chem. Phys. 2010, 132, 154104 .

(34) Gaus, M.; Goez, A.; Elstner, M. Parametrization and Benchmark of DFTB3 for Organic Molecules. J. Chem. Theory Comput. 2013, 9, 338.

(35) Gaus, M.; Lu, X.; Elstner, M.; Cui, Q. Parameterization of DFTB3/3OB for Sulfur and Phosphorus for Chemical and Biological Applications. J. Chem. Theory Comput. 2014, 10, 1518.

(36) Monkhorst, H. J.; Pack, J. D. Special Points for Brillouin-Zone integrations. Phys. Rev. B 1976, 13, 5188. 
(37) Martínez, J. M.; Martínez, L. Packing optimization for automated generation of complex system's initial configurations for molecular dynamics and docking. J. Comput. Chem. 2003, 24, 819.

(38) Martínez, L.; Andrade, R.; Birgin, E. G.; Martínez, J. M. Packmol: A package for building initial configurations for molecular dynamics simulations. J. Comput. Chem. 2009, 30, 2157.

(39) Adhikari, B.; Trieu, T.; Cheng, J. Chromosome3D: reconstructing threedimensional chromosomal structures from Hi-C interaction frequency data using distance geometry simulated annealing. BMC Genom. 2016, 17, 886.

(40) Andersen, H. C. Molecular dynamics simulations at constant pressure and/or temperature. J. Chem. Phys. 1980, 72, 2384.

(41) Aradi, B.; Hourahine, B.; Frauenheim, T. DFTB+, A sparse Matrix-Based Implementation of the DFTB Method. J. Phys. Chem. A 2007, 111, 5678.

(42) Chen, X.; Tan, C.; Yang, Q.; Meng, R.; Liang, Q.; Jiang, J.; Sun, X.; Yang, D. Q.; Ren, T. Effect of multilayer structure, stacking order and external electric field on the electrical properties of few-layer boron-phosphide. Phys. Chem. Chem. Phys. 2016, 18, 16229-16236.

(43) Gustinčič, D.; Kokalj, A. A DFT study of adsorption of imidazole, triazole, and tetrazole on oxidized copper surfaces: $\mathrm{Cu} 2 \mathrm{O}(111)$ and $\mathrm{Cu} 2 \mathrm{O}(111)-\mathrm{w} / \mathrm{o}-\mathrm{CuCUS}$. Phys. Chem. Chem. Phys. 2015, 17, 28602.

(44) Guterman, T.; Gazit, E. Toward peptidebased bioelectronics: reductionist design of conductive pili mimetics. Bioelectron. Med. 2018, 1, 131.

(45) Tulip, P. R.; Clark, S. J. Structural and electronic properties of L-amino acids. Phys. Rev. B 2005, 71, 195117. 


\section{Graphical TOC Entry}

Some journals require a graphical entry for the Table of Contents. This should be laid out "print ready" so that the sizing of the text is correct. Inside the tocentry environment, the font used is Helvetica $8 \mathrm{pt}$, as required by Journal of the American Chemical Society.

The surrounding frame is $9 \mathrm{~cm}$ by $3.5 \mathrm{~cm}$, which is the maximum permitted for Journal of the American Chemical Society graphical table of content entries. The box will not resize if the content is too big: instead it will overflow the edge of the box.

This box and the associated title will always be printed on a separate page at the end of the document. 